\title{
Construção de serious games para adolescentes com diabetes mellitus tipo 1
}

\author{
Construction of serious games for adolescents with type 1 diabetes mellitus
}

Elaboración de serious games para adolescentes con diabetes mellitus tipo 1

Andréia Régia Rodrigues de Matos Serafim 1

Amanda Newle Sousa Silva ${ }^{1}$

Caroline Magalhães de Alcântara ${ }^{1}$

Maria Veraci Oliveira Queiroz ${ }^{1}$

\section{Descritores}

Enfermagem pediátrica; Diabetes mellitus tipo 1; Jogos de vídeo; Adolescentes; Educação em saúde

\section{Keywords}

Pediatric nursing; Diabetes mellitus, type 1; Video games; Adolescent; Health education

\section{Descriptores}

Enfermería pediátrica; Diabetes mellitus tipo 1;

Juegos de vídeo; Adolescente; Educación en salud

\section{Submetido}

30 de Junho de 2018

Aceito

2 de Maio de 2019

\section{Resumo}

Objetivo: Construir serious games educativo para adolescentes com diabetes mellitus tipo 1.

Métodos: Pesquisa metodológica, fundamentado no design centrado no usuário e efetivado em três etapas: revisão de literatura e envolvimento dos sujeitos; concepção e produção do protótipo; avaliação da usabilidade.

Resultados: a produção do serious games foi pautada em literatura científica e sugestões de adolescentes. 0 conteúdo educativo foi apresentado pelo personagem Didi sobre hábitos de vida diária: acordar, realizar higiene pessoal, monitorização da glicemia capilar e aplicação de insulina. Neste percurso, o jogador observa ações terapêuticas incluindo escolhas de alimentos saudáveis. Na avaliação de usabilidade, os adolescentes consideraram satisfatórios os requisitos de jogabilidade, conteúdo e design.

Conclusão: 0 teste de usabilidade teve validação positiva e as sugestões contribuíram para melhoria da qualidade das funções e interação, favorecendo o alcance das metas do jogo. Assim, a tecnologia apresenta propriedades para melhorar os cuidados dos adolescentes com diabetes mellitus tipo 1.

\section{Abstract}

Objective: To construct serious educational games for adolescents with type 1 diabetes mellitus.

Methods: Methodological research, based on patient-centered design, conducted in three steps: literature review and subjects' involvement; design and production of the prototype; usability assessment.

Results: The production of serious games was based on scientific literature and suggestions of adolescents. The educational content was presented by the Didi character, about habits of daily living: waking up, personal hygiene, monitoring of capillary glycemia, and insulin administration. In this sequence, the player observes therapeutic actions, including healthy food choices. In the usability assessment, the adolescents considered the requirements of gameplay, content, and design to be satisfying.

Conclusion: The usability test resulted in positive validation, and the suggestions contributed to improvement of the quality of the functions and interactions, favoring the achievement of the goals of the game. Thus, the technology has properties to improve the care of adolescents with type 1 diabetes mellitus.

\section{Resumen}

Objetivo: construir serious games educativos para adolescentes con diabetes mellitus tipo 1.

Métodos: investigación metodológica, con fundamento en el diseño centrado en el usuario y realizado en tres etapas: revisión de la literatura y participación de los sujetos, concepción y producción de prototipo, evaluación de usabilidad.

Resultados: la producción del serious game se realizó de acuerdo con literatura científica y sugerencias de adolescentes. El contenido educativo fue presentado por el personaje Didi sobre hábitos de la vida cotidiana: despertarse, realizar aseo personal, monitoreo de glucemia capilar y aplicación de insulina. En ese trayecto, el jugador observa acciones terapéuticas, que incluye la elección de alimentos saludables. En la evaluación de usabilidad, los adolescentes consideraron satisfactorios los requisitos de jugabilidad, contenido y diseño.

Conclusión: la prueba de usabilidad tuvo validación positiva y las sugerencias contribuyeron para mejorar la calidad de las funciones de interacción, lo que favoreció el cumplimiento de los objetivos del juego. De esta forma, la tecnología presenta propiedades para mejorar los cuidados de los adolescentes con diabetes mellitus tipo 1.

\section{Autor correspondente}

Amanda Newle Sousa Silva

http://orcid.org/0000-0001-5728-847X

E-mail: amanda.newle@aluno.uece.br

\section{DOI}

http://dx.doi.org/10.1590/1982-

0194201900052

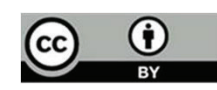

\footnotetext{
Como citar:

Serafim AR, Silva NA, Alcântara CM, Queiroz MV. Construção de serious games para adolescentes com diabetes mellitus tipo 1. Acta Paul Enferm. 2019;32(4):374-81.
} 


\section{Introdução}

O diabetes mellitus tipo 1 é uma das doenças crônicas mais comuns na infância, cuja incidência continua a aumentar em todo o mundo. ${ }^{(1-3)}$ Entre os países com maior número de casos, destacam-se os Estados Unidos da América, a Índia e o Brasil, em terceiro lugar. ${ }^{(3,4)}$

Há impactos na vida social e econômica provocados pelo diabetes, ${ }^{(5)}$ e a vivência dos adolescentes com DM1 é permeada de enfrentamentos e dificuldades pelas restriçóes nos hábitos e na rotina de cuidados intensos, incluindo um plano sobre dieta, testes glicêmicos, insulinoterapia que, às vezes, limita a vida social. ${ }^{(6)}$

Os cuidados são orientados pela equipe de saúde especializada e envolvem intervençôes educativas que devem motivar a adesáo terapêutica e ajudar o adolescente a enfrentar o desafio do controle metabólico. ${ }^{(7)}$ Contudo, a revisão de literatura aponta os tipos de tecnologias produzidas para educação em saúde do adolescente com DM1, mas na proposta de tecnologia digital integrando os principais cuidados não foram encontradas evidências científicas no âmbito nacional.

Desse modo, a aprendizagem deve ser significativa, ou seja, incluir conteúdos que somam aos conhecimentos prévios dos adolescentes e assim, favorecer reflexôes e comportamentos saudáveis com autonomia e independência. ${ }^{(8)}$ Destarte, para atingir esse público, é importante criar estratégias interativas e motivadoras que promovam informaçóes de saúde sobre o cuidado diário.

Nesse sentido, a expansão das tecnologias da informação possibilitou que a internet, os telefones celulares, redes sociais (Twitter e Facebook), jogos eletrônicos, programas de educação online, tablets e outros dispositivos incluíssem os adolescentes como principais utilizadores destas tecnologias de comunicação. ${ }^{(9,10)}$ E, no contexto digital, o serious games, entendido como jogo sério e voltado para educação em saúde $^{(11)}$ e pode proporcionar aos adolescentes com DM1 incentivos aos cuidados diários, uma vez que estas tecnologias estão presentes em seu cotidiano. ${ }^{\left({ }^{9}\right)}$ Portanto, ferramentas de utilidade à prática educativa em situaçóes de adoecimento crônico.
Vale salientar que o jogo é um espaço para aprendizagem e proporciona atividade lúdica, atrativa e inovadora. O serious games caracteriza-se por permitir a integração do sujeito ao jogo em uma perspectiva educativa, estimulando os adolescentes nos cuidados à saúde. ${ }^{(9,11,12)}$ Outra particularidade do jogo é o envolvimento dos jovens na busca por descobrir o mundo virtual. ${ }^{(13)}$ Além disso, favorecem atividades de prazer e inclusão social. ${ }^{(10)}$ Promovem o elo entre brincar e aprender, permitindo aprendizagem de regras e incentivando os cuidados à saúde. ${ }^{(11,13)}$

Nessa perspectiva, foi elaborado um serious game com propósito de favorecer uma aprendizagem significativa, ${ }^{(8)}$ despertando interesse e envolvimento de adolescentes ao cuidado à saúde. Ante ao exposto, o estudo teve como objetivo construir serious games educativo para adolescentes com diabetes mellitus tipo 1.

\section{Métodos}

Pesquisa metodológica, que investiga, organiza e analisa dados para construir, validar instrumentos e técnicas de pesquisa. ${ }^{(14)} \mathrm{A}$ construção do jogo foi fundamentado em referência pedagógica da aprendizagem significativa ${ }^{(8)}$ e design centrado no usuário, ${ }^{(15)}$ e sua realização se deu entre janeiro a maio de 2018, cujas três etapas de construção foram: revisão de literatura e envolvimento dos usuários em pesquisa de campo; concepção e produção; teste de usabilidade para refinamento do protótipo.

$\mathrm{Na}$ primeira etapa, o estudo de revisão foi norteado pela questão: que tecnologias educativas são desenvolvidas para adolescentes com diabetes mellitus tipo 1? Buscou-se nas bases de dados National Library of Medicine National Institutes of Health (PUBMED), Cumulative Index to Nursing and Allied Health Literature (CINAHL) e na Base de dados em Enfermagem (BDENF), utilizando os descritores enfermagem pediátrica, diabetes mellitus tipo 1, jogos de vídeo, adolescentes e educação em saúde, resultando em 14 artigos, os quais sinalizaram os tipos de tecnologias produzidas para educação em saúde do adolescente com DM1 e a lacuna em tecnologias com jogos sérios. Os resultados desta revisão, 
os manuais da Sociedade Brasileira de Diabetes ${ }^{(4)} \mathrm{e}$ American Association of Diabetes Educators, ${ }^{(16)}$ contribuíram na concepção e elaboração do conteúdo visando incluir cuidados específicos ao adolescente com DM1.

A pesquisa de campo para apreender as necessidades e envolver os usuários ocorreu em centro de referência no atendimento aos pacientes com DM1. No caso, com 16 adolescentes de 10 a 19 anos e seis profissionais da assistência sendo um médico, um nutricionista, um fisioterapeuta e três enfermeiros, estes responderam um roteiro semi-estruturado que contemplava necessidades e interesses, uso de tecnologias digitais e sugestóes sobre a apresentação (design) da possível tecnologia como cores, linguagem e sons.

A segunda etapa foi desenvolvido o plano de criação, a pré-produção, a partir da análise das etapas anteriores: literatura revisada e envolvimento de usuários e profissionais. ${ }^{(15,17)} \mathrm{O}$ protótipo do jogo foi realizado em um modelo de software, utilizando simulaçóes e testes para aperfeiçoamento, assegurando boa jogabilidade.

$\mathrm{Na}$ terceira etapa para o teste de usabilidade de forma interativa foi realizada adequação e aperfeiçoamento do jogo, ${ }^{(18,19)}$ aplicado com cinco adolescentes entre 12 e 17 anos, escolhidos por conveniência, realizada no mesmo centro de referência para atendimento aos pacientes com diabetes em FortalezaCE, Brasil.

A avaliação da usabilidade envolveu esses adolescentes na jogabilidade e o teste foi aplicado na sala de espera, individualmente, momento em que foi apresentado no computador o jogo e os comandos para utilização. Cada adolescente teve interação com a tecnologia em média 20 minutos. Ao concluírem esse momento foi aplicado um questionário, que continham itens e suas pontuaçóes, para saber se o jogo atendeu ao propósito, efetivação da jogabilidade e sugestôes para melhoria.

O questionário para análise da usabilidade foi adaptado $^{(20)}$ e contemplavam os itens sobre atenção, relevância, confiança e satisfação, buscando-se observar os estímulos e de certo modo, a motivação despertada pelo jogo educativo, a expectativa do usuário em relação à tecnologia, ao engajamento e à jogabilidade. Atribuíram-se notas aplicadas em uma escala de Likert, com valoração de 1 a 5 (insatisfatório) e de 6 a 10 (satisfatório).

As sugestóes indicadas pelos adolescentes durante a avaliação da usabilidade do jogo foram incorporadas à tecnologia educativa, com auxílio de um profissional de informática que realizou as alteraçôes propostas. Com isso, efetivou-se o reparo e aprimoramento das disfunçóes observadas.

O estudo foi aprovado pelo Comitê de Ética em Pesquisa da Universidade Estadual do Ceará, recebendo o CAAE 74169617.7.0000 e parecer $\mathrm{n}^{\mathrm{o}}$ 2.468.749. A investigação seguiu os preceitos éticos da pesquisa com seres humanos e o Termo de Assentimento Livre Esclarecido foi apreciado e assinado pelos adolescentes e o Termo de Consentimento Livre e Esclarecido pelos responsáveis, em todas as fases que tiveram a participação dos sujeitos. $^{(21)}$

\section{Resultados}

O desenvolvimento do serious games está apresentado descritivamente desde a sua idealização e construção à usabilidade realizada com questionamentos aos adolescentes que foram compilados com as notas individuais e a média para confirmação da validação do teste.

Na primeira etapa do estudo, todos os artigos no âmbito internacional mostraram benefícios no uso da tecnologia para a educação de adolescentes com DM1. Identificou-se programas de aconselhamento, sistemas de mensagens de texto, fóruns, blogs, ferramentas de redes sociais, aplicativos, programas de treinamento online com focos específicos como: educação reprodutiva, controle glicêmico, alimentação, insulinoterapia, resolução na autogestão e habilidades de enfrentamento do diabetes. Contudo, nenhum desses estudos, no escopo planejado, foi localizado no âmbito brasileiro.

Os adolescentes ressaltaram o interesse pelo uso das tecnologias digitais, aspectos presentes em seu cotidiano, quais sejam: os jogos digitais e o seu acesso, por meio de smartfone, tablet e computadores, importância de materiais ilustrativos e frases curtas. 
Os profissionais sinalizaram as vantagens do uso da tecnologia, pois atendem aspectos lúdicos de aprendizagem e estáo presentes no cotidiano deste público. Mencionaram o respeito à linguagem, apresentação dinâmica, com cores fortes e sons para motivação dos adolescentes aos cuidados diários.

$\mathrm{Na}$ segunda etapa o planejamento do protótipo, os pesquisadores reuniram-se para traçar estratégias, discussão de conceitos, agregando as informaçóes da literatura revisada e da pesquisa de campo. Foram oito reunióes com os profissionais da tecnologia da informação e com o desing gráfico, para que esses compreendessem os conteúdos e o contexto a ser alcançado na construção tecnológica.

As fases operacionais de construção da tecnologia consistiram em elaboração do Game Design Document, relatório detalhado, conceituação artística, jogabilidade e definiçóes da interface do jogo. Os conteúdos abordados no serious games, denominado "Batalha do Didi", tinham como finalidade despertar no jogador a autonomia nos cuidados, por meio do mundo virtual, associada à vivência no mundo real.

O personagem principal denominado Didi foi o mesmo constante no website (www.diabetesemfoco.com), desenvolvido pelo Grupo de Pesquisa Cuidado à Saúde da Criança e do Adolescente e Enfermagem (GEPCCA).

A interface de apresentação do jogo, exposta na figura 1, tem quatro opçôes: jogar, ranking (com pontuaçóes de cada jogador), ajuda (apresenta os objetivos do jogo e tutorial) e o reiniciar. $\mathrm{O}$ jogo educativo é compatível com androide e windows, formato de dimensão (2D), utilizando a engine Game Make Studio 1.4. Para o funcionamento do jogo e interação usuário e computador, optou-se pelo uso do teclado.

$\mathrm{O}$ serious games constituiu-se de cinco fases e se inicia com o personagem Didi acordando e realizando suas atividades de vida diária, predispondo-se às escolhas para atividades de autocuidado. Seguidamente, na segunda fase, o adolescente verifica a glicemia capilar, realiza a aplicação da insulina e faz a refeição da manhã com alimentos saudáveis. Ao final desta fase, o Didi encontra-se com a família que representa o suporte social primário ao sujeito adoecido.

$\mathrm{Na}$ terceira fase do jogo, o Didi pratica uma atividade física e toma decisóes na oferta dos alimentos

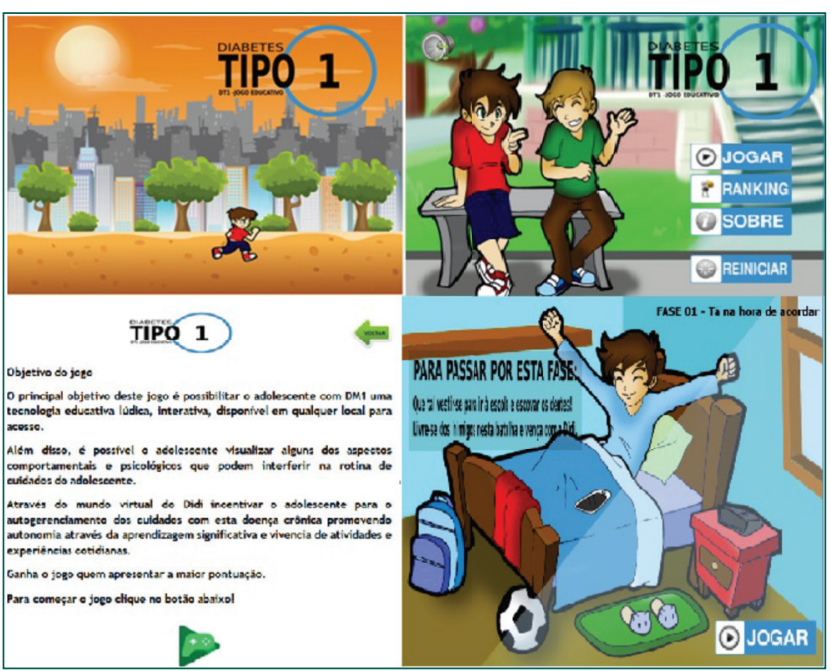

Figura 1. Apresentação do serius game "Batalha do Didi"

saudáveis ou não; o adolescente vai realizar escolhas, nas quais, optando por alimentos nutritivos, ganha pontos. $\mathrm{Na}$ quarta fase, ao término da aula, Didi volta para casa e vai ao mercado. Neste momento, ele faz escolhas de alimentos que vai compor sua dieta, estimulando suas preferências alimentares, ganhando pontos com as escolhas saudáveis. $\mathrm{Na}$ quinta fase é hora de voltar para casa, então, ele anda de skate e leva alguns alimentos saudáveis para consumir posteriormente.

Nestas fases, sintetizados na figura 2, foram inseridos obstáculos e dificuldades vivenciados pelos adolescentes com DM1 na rotina diária de cuidados, os quais são representados pelos personagens (avatares coadjuvantes), ou seja, a "desmotivação" e a "tristeza", sendo necessário que o Didi desvie destas barreiras e ultrapasse esses agentes que, muitas vezes, enfrentam no mundo real frente ao tratamento e cuidados diários. Caso o jogador opte por ignorar os obstáculos virtuais e enfrente com Didi, as barreiras impostas pela "desmotivação" e "tristeza", o jogador perde pontos, podendo chegar a game over, o que significa literalmente, fim de jogo, isto é, não conseguiu concluir o desafio proposto.

Vale salientar que o mundo virtual está representando os desafios vivenciados por adolescentes com DM1, mas que no cotidiano, eles precisam enfrentar e superar com o apoio da rede social, incluindo a família e os profissionais da saúde.

O serious games, também, comportou recursos sonoros de alerta, como o despertador ao acordar, o 


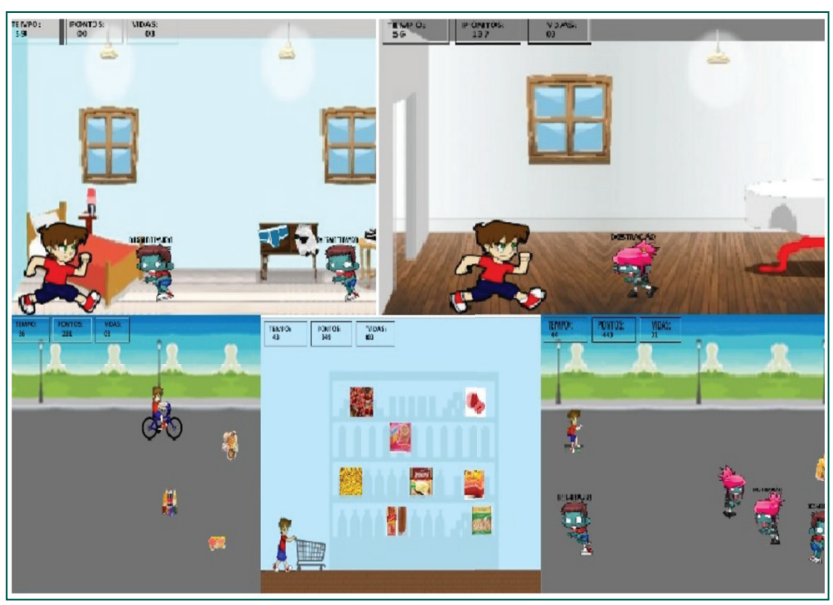

Figura 2. Fases do serious game: "Batalha do Didi"

comando de voz de dublagem, para que o jogador possa compreender as regras e a operacionalizaçáo. Foi incluída música para motivar o momento em que Didi realiza atividade física, como pedalar de bicicleta e andar de skate, possibilitando o jogador simulação do mundo real.

As imagens utilizadas foram de autoria própria, elaboradas por um designer gráfico, juntamente com outros profissionais especialistas que compóem o grupo de pesquisadores mencionados. O propósito foi desenvolver ambientes alegres, cores fortes e vibrantes, cenários motivadores, lúdicos e familiares. As atividades físicas foram praticadas pelo Didi em cenários urbanos.

Em relação ao teste de usabilidade apresentaramse os critérios de avaliação de jogos educacionais, sendo utilizadas as seguintes dimensóes: atenção, relevância, confiança e satisfação. Os adolescentes emitiram sugestóes, dentre estas, aprimorar as ilustraçóes da última fase, pois o tamanho das figuras estava pequeno, isso dificultava a vizualização e a escolha dos alimentos inseridos no plano alimentar. Ao final, no quarto estágio, após os testes de usabilidade, efetivouse a fase de reparo, com todas as sugestóes acatadas e modificadas pelo programador, participante da equipe de construção do serious games.

\section{Discussão}

Visualiza-se limites dos resultados, relacionado ao número de adolescentes que participaram do teste de jogabilidade e por ter ocorrido na sala de espera das consultas, a disponibilidade dos mesmos ficava reduzida, pois o seu familiar demonstrava pressa na conclusão da tarefa. Contudo, os adolescentes faziam imersão na proposta de jogabilidade, trazendo, portanto confirmação do interesse pelo jogo.

Desta forma, observa-se a possiblidade de contribuição na prática dos enfermeiros, especialmente, no desenvolvimento de intervençôes educativas envolvendo adolescentes com DM1. Vislumbra-se a continuidade da validação do referido jogo para que o mesmo aumente a confiabilidade e a efetividade em açóes educativas, permitindo sua incorporação no cuidado clínico do enfermeiro.

$\mathrm{O}$ roteiro ou narrativa do jogo apresentava simulação do cotidiano de adolescentes com DM1, incentivando-os a tomar decisóes no mundo virtual, observando a rotina dos cuidados e comportamentos de autocuidado. ${ }^{(16)} \mathrm{O}$ serious games como tecnologia digital constitui-se de um recurso lúdico, que confere descontração, interatividade e possível ação educativa, considerando sua aceitação e utilização cada vez mais presente no cotidiano dos adolescentes, portanto facilitadora de cuidados na condição de DM1. Para o seu uso é necessário computador, smartfone ou ferramenta similar.

A tecnologia buscou disponibilizar educação em saúde por meio da web. Esta escolha referente ao nome do jogo "Batalha do Didi", aconteceu durante a pesquisa de campo com os adolescentes, buscando-se representar os desafios diários, interligando a identidade virtual ficcional com o mundo real vivido na condição de adoecimento. ${ }^{(22)}$

A literatura aponta que o serious games imprime qualidades virtuais que despertam interesse e adesão do adolescente, de forma que ele aprenda e se divirta simultaneamente. ${ }^{(12,13)} \mathrm{Na}$ atualidade, eles convivem com uma infinidade de informaçóes e recursos tecnológicos, que os tornam cada vez mais autônomos e participativos nos processos educativos. ${ }^{(13)}$ Recomenda-se, portanto, envolve-los desde a fase de elaboração e nos processo de funcionalidade, ampliando-se a possibilidades de alcance dos resultados pretendidos. ${ }^{(15)}$

$\mathrm{O}$ jogo produzido permite aos jogadores alcançar pontos como recompensa e interação, de 
modo simples e natural, associando-as a rotina de cuidados e atendendo assim, a finalidade do mesmo. As tecnologias educativas são necessárias para facilitar o ensino da enfermagem e a prática do cuidar, pois eleva o nível de conhecimento e motiva o cuidado. ${ }^{(11,23)}$ Desse modo, abordaramse no serious games os sete principais comportamentos de autocuidado da American Association of Diabetes Educators: alimentação saudável, monitorização glicêmica, uso da medicação com segurança, atividade física, resolução de problemas, enfrentamento saudável e redução de riscos. ${ }^{(4,16)}$

Nesta perspectiva, demostram-se os desafios representados pelos avatares coaduvantes simbolizando os sentimentos de desmotivação e tristeza, que são apontados na literatura. ${ }^{(4,24)}$ Estas reaçóes comprometem a adesão terapêutica e a qualidade de vida dos sujeitos adoecidos. O modo de recompensa no jogo educativo está nas escolhas certas pautadas nos conhecimentos voltados à adesão ao tratamento, ganhando pontos e motivando o participante a vencer cada fase e finalizar o jogo.

Assim, as tecnologias digitais, como o serious games, são consideradas meios de proporcionar educação em saúde, estratégia que pode estimular os cuidados, pois são, muitas vezes, desafiadoras para os adolescentes. ${ }^{(20,22,25,26)}$ A tecnologia construída contempla atividades da vida diária dos adolescentes, com práticas comuns a todos, como vestir-se, alimentar-se, fazer atividade física e ir à escola. A participação dos profissionais da assistência ajudou na elaboração e facilitou a adequação do conteúdo, aproximando-se do cotidiano do usuário final. ${ }^{(27)}$

O estudo demonstrou as atividades de cuidados diários de adolescentes com DM1 em decorrência do tratamento contínuo que, muitas vezes, se inicia na infância. E para entender a necessidade de cumprir a rotina diária e evitar descompensaçóes agudas, como a cetoacidose diabética, dentre outras, os profissionais da saúde e a família podem colaborar, substancialmente, para que esse adolescente assuma o próprio cuidado e tenha uma vida saudável tão próxima quanto possível daqueles que não têm diabetes. ${ }^{(15)}$

A primeira e segunda fase do jogo retratam o autogerenciamento de cuidados diários como hábitos higiênicos, vestir-se, alimentar-se e ir ao colé- gio, este como indispensável ao desenvolvimento do adolescente. ${ }^{(27)} \mathrm{O}$ jogo traz estímulos ao adolescente na tomada da insulina e na verificação glicêmica, pois a cada ação terapêutica, ele acumula pontos e isso os motivará a alcançar responsabilidade e maior autonomia nos cuidados. ${ }^{(25)}$

Essas atividades, incluindo a alimentação saudável e reposição insulínica, são fundamentais para o controle metabólico. Este controle terapêutico bloqueia a lipólise e a produção hepática de glicose, enquanto períodos sem insulina são causas de oscilaçóes glicêmicas amplas, acrescentando fator de resistência à insulina proporcionada pela própria hiperglicemia. ${ }^{(4)}$ Portanto, estes aspectos foram estimulados no jogo como prioridade no cuidado ao adolescente com DM1.

Outro aspecto ressaltado para o tratamento foi a realização de atividade física, andar de bicicleta e skate também foram demonstrados, pois são esportes comuns nessa faixa etária, e indicados ao DM1. A prática de atividade física regular reduz a resistência à insulina, facilitando a utilização periférica de glicose e contribuindo para a melhoria do controle glicêmico. ${ }^{(4)}$

$\mathrm{Na}$ quarta fase, o adolescente com DM1 é estimulado a fazer escolhas alimentares, uma necessidade do tratamento. ${ }^{(23)} \mathrm{Na}$ presente pesquisa isso é demonstrado quando ele vai ao mercado e coloca no carrinho alimentos desejados. Em outro estudo salienta-se que, apesar dos adolescentes terem ideia do que é uma alimentação saudável, eles têm dificuldade de aceitar as restriçóes, mesmo sabendo das consequências. Pesquisa aponta que fazem associação ao conhecimento sobre alimentação saudável com o que é exposto na escola, e que, essa prática está vinculada à qualidade vida. Entretanto, muitos ainda persistem na ingestão de lanches não nutritivos, como fast food e guloseimas. A mudança do comportamento alimentar náo é simples, atitude que requer tempo, exige planejamento e implementação de práticas educativas contínuas, principalmente no ambiente escolar, onde passam a maior parte do tempo. ${ }^{(28)}$

Para representar as dificuldades enfrentadas diariamente foram utilizados os avatares como simbologia, representados pela "desmotivação e tristeza" ${ }^{(23)}$ vivenciadas, principalmente, no início do tratamento, mas que são superados ao aprender a conviver com 
algumas limitaçóes e assumir os cuidados indispensáveis ao controle metabólico. Ademais, pontuam-se outros sentimentos negativos, como o medo e a insegurança em torno de uma nova realidade, permeadas de restriçóes na dieta, limitação física e cobrança dos cuidadores. ${ }^{(28)} \mathrm{O}$ jogo por ser uma atividade lúdica e de descobertas que integra o sujeito entre o mundo virtual e o real pode impulsionar os adolescentes a assumirem os cuidados à saúde. ${ }^{(11,12)}$

A participação do público-alvo contribuiu desde a concepção até o teste de jogabilidade permitindo contemplar as necessidades dos adolescentes, fazendo associação a sua finalidade, estratégia de ensino baseada em aprendizagem significativa, buscando promover mais que uma aprendizagem mecânica ou memorística, ela reelabora conhecimentos prévios e propóe conhecimentos complementares a este público. ${ }^{(8)}$ Essa tecnologia, além de ser uma estratégia criativa, pode sensibilizar o adolescente a participar dos próprios cuidados, fundamentado na motivaçáo, no contexto, na interatividade e no dinamismo das informaçôes, propondo momentos de descontração e reflexão. ${ }^{(14)}$

$\mathrm{Na}$ avaliação da usabilidade, os adolescentes julgaram as dimensóes avaliadas como satisfatórias e os índices de concordância que qualificam o jogo como uma interface amigável e de uso descomplicado, ou seja, o produto tecnológico, na visão dos adolescentes, sinaliza a usabilidade adequada ao público a que se destina. Consideraram-se, portanto, as avaliaçóes positivas, segundo a percepçáo dos adolescentes, quando experimentaram jogar de forma lúdica e atingiram as expectativas impostas pelo desafio do jogo. ${ }^{(29)}$

Após análise dos dados da avaliação de usabilidade, confirmou-se a pertinência em utilizá-la como ferramenta educativa, pois os desafios propostos correspondem a um dos aspectos mais importantes em jogos com esta finalidade. Ele precisa ser suficientemente desafiador, estar adequado ao nível cognitivo do jogador, exigindo níveis crescentes de destreza e habilidade. ${ }^{(16,20)}$

Ademais, as ilustraçóes são estratégias utilizadas em tecnologias digitais para descrever o conteúdo e induzirem significados a fim de favorecer o aprendizado. ${ }^{(30)}$ Os adolescentes avaliaram satisfatórias as imagens e o cenário aplicados na dinâmica do jogo.
É inegável que as açóes educativas desenvolvidas pelos profissionais de saúde com os adolescentes na condição de DM1, devam ser facilitadas por tecnologias educativas que envolvam estes sujeitos. Os enfermeiros são capacitados para atuar através da conduta terapêutica com os pacientes, fornecendo educação e apoio. ${ }^{(31)} \mathrm{O}$ desenvolvimento do jogo partiu de conteúdos encontrados na literatura, experiência prévia em estudo anterior e avaliaçáo feita pelos adolescentes sobre a usabilidade do jogo. Estas etapas desenvolvidas conjugadamente, permitiram inferir que a tecnologia como proposta educativa, expressa os conhecimentos prévios dos adolescentes e desperta o ânimo, encorajando-o nos cuidados contínuos à sua saúde.

\section{Conclusão}

A criação do serious games foi efetivada, associando experiências e conhecimentos sobre os cuidados de adolescentes na condição de DM1, traz, portanto, o cotidiano dos mesmos, ou seja, situaçóes do mundo real contextualizadas no mundo virtual com vivências e enfrentamentos no percurso dos cuidados diários. Esta participação, mesmo com poucos sujeitos, concretizou-se também no teste de jogabilidade, propicinando além da interação melhoria nos propósitos do jogo. Desse modo, os adolescentes julgaram positiva a proposta do jogo educativo nos itens observados, demonstrando fácil utilização, expuseram que as regras estavam claras e que a apresentação gráfica era motivadora e interativa. Concluiu-se que o teste de usabilidade teve resposta positivas pelos adolescentes e as sugestóes contribuíram para melhoria da qualidade das funçóes e interação, favorecendo o alcance das metas do jogo. Assim, a tecnologia apresenta propriedades para melhorar os cuidados dos adolescentes com DM1.

\section{Agradecimentos}

Agradecemos o apoio da Universidade Estadual do Ceará pelo espaço e suporte nos equipamentos como computadores para elaboração do serious games, além dos profissionais da Tecnologia da Informação 
pela programação do artefato tecnológico. A pesquisa não recebeu financiamento para a sua realização.

\section{Colaborações}

\section{Serafim ARRM, Silva ANS, Alcântara CM e Queiroz MVO declaram que contribuíram com a concepção do estudo, análise e interpretação dos dados, redação do artigo, revisão crítica relevante do conteúdo intelectual e aprovação da versão final a ser publicada.}

\section{Referências}

1. Atkinson MA, Eisenbarth GS, Michels AW. Type 1 diabetes. Lancet. 2014;383(9911):69-82.

2. Isla Pera P, Palacin Lois M, López Matheu C, Honrubia Pérez M, Gómez Rodriguez AM, Armengol Camps E, et al. Perceptions of diabetes obtained through drawing in childhood and adolescence. Patient Prefer Adherence. 2013;7:595-605.

3. Patterson C, Guariguata L, Dahlquist G, Soltész G, Ogle G, Silink M. Diabetes in the young - a global view and worldwide estimates of numbers of children with type 1 diabetes. Diabetes Res Clin Pract. 2014;103(2):161-75.

4. Sociedade Brasileira de Diabetes. Diretrizes da Sociedade Brasileira de Diabetes: 2015-2016. São Paulo: A. C. Farmacêutica; 2016.

5. Organização Mundial de Saúde. Cuidados inovadores para condições crônicas: organização e prestação de atenção de alta qualidade às doenças crônicas não transmissíveis nas Américas. Washington (DC): OMS; 2015.

6. Pennafort VP, Silva AN, Queiroz MV. [The perception of nurses regarding educational practices for children with diabetes in hospital care]. Rev Gaúcha Enferm. 2014;35(3):130-6. Portuguese.

7. King KM, King PJ, Nayar R, Wilkes S. Perceptions of Adolescent Patients of the "Lived Experience" of Type 1 Diabetes. Diabetes Spectr. 2017;30(1):23-35.

8. Moreira MA. Al final, que és aprendizaje significativo? Rev Qurriculum. 2012;25:29-56.

9. Paul L. Improving health in adolescents with the use of information technologies. Online J Nurs Inform. 2012;6(1):1-12.

10. Carrion C, Arroyo M, L., Castell C, Puigdomènech E, Gómez S F, Domingo L, Espallargues M. Utilización del teléfono móvil para el fomento de hábitos saludables en adolescentes. Rev Esp Salud Publica. 2016; 90(Esp): 1-11.

11. Deguirmendjian S, Miranda FM, Zem-Mascarenhas SH. Serious game desenvolvidos na saúde: revisão integrativa da literatura. J Health Inform. 2016;8(3):110-6.

12. Fraticelli F, Marchetti D, Polcini F, Mohn AA, Chiarelli F, Fulcheri M, et al. Technology-based intervention for healthy lifestyle promotion in Italian adolescents. Ann Ist Super Sanita. 2016;52(1):123-7.
13. Cavalli FD, Trevisol MT, Vendrame T. Influência dos jogos eletrônicos e virtuais no comportamento social dos adolescentes. Psicol Argum. 2013;31(72):155-63.

14. Polit DF, Beck CT. Fundamentos de pesquisa em enfermagem: avaliação de evidências para a prática da enfermagem. 7th ed. Porto Alegre: Artmed; 2011.

15. De Vito Dabbs A, Myers BA, Mc Curry KR, Dunbar-Jacob J, Hawkins RP, Begey A, et al. User-centered design and interactive health technologies for patients. Comput Inform Nurs. 2009;27(3):175-83.

16. Pera MP, Lois PM, Matheu LC, Pe $\neg$ rez MH, Rodr $f \pm$ guez AG, Camps $E A$, et al. Perceptions of diabetes obtained through drawing in childhood and adolescence. Patient Prefer Adher. 2013;(7):595-605.

17. Savi R, Wangenheim CGV, Ulbricht V, Vanzin T. Proposta de um modelo de avaliação de jogos educacionais. RENOTE. 2010; 8(3):1-13.

18. Machado LD, Ronei M, Nunes FL, Costa RM. Serious games baseados em realidade virtual para educação médica. Rev Bras Educ Med. 2011;35(2):254-62.

19. Lynn MR. Determination and quantification of content validity. Nurs Res. 1986;35(6):382-5.

20. Carvalho LR, Évora YD, Zem-Mascarenhas SH. Avaliação de usabilidade de um protótipo de tecnologia digital educacional sobre monitoração da pressão intracraniana. Ver Lat Am Enfermagem. 2016;24:e2777.

21. Brasil. Ministério da Saúde. Conselho Nacional de Saúde. Diretrizes e normas regulamentadoras de pesquisa envolvendo seres humanos. Resolução n. 466, de 12 de dezembro de 2012. Brasília (DF): Ministério da Saúde; 2012.

22. Trepte S, Reinecke L. Avatar creation and video game enjoyment: effects of life-satisfaction, game competitiveness, and identification with the avatar. J Media Psychol. 2010;22(4):171-84.

23. Andrade CJ, Alves CD. Fatores associados ao controle glicêmico em crianças e adolescentes com diabetes melito tipo 1. R Ci Med Biol. 2012;11(2):234-8.

24. Alencar DC, Lima AC, de Almeida VC, Sampaio KJ, Damasceno MM, Alencar AM. Sentimentos de adolescentes com diabetes mellitus frente ao processo de viver com a doença. Rev Bras Enferm. 2013;66(4):479-84.

25. Sales-Peres SH, Guedes MF, Sá LM, Negrato CA, Lauris JR. Estilo de vida em pacientes portadores de diabetes mellitus tipo 1: uma revisão sistemática. Ciênc Saúde Coletiva. 2016; 21(4): 1197-206.

26. Adams J. Life experience for an adolescent with type 1 diabetes: nursing strategies to support a healthy lifestyle. Whitireia Nurs Health J. 2012; (19):18-26.

27. Machado LD, Moraes RM, Nunes FL. Serious games para saúde e treinamento imersivo: abordagens práticas de realidade virtual e aumentada. Porto Alegre: SBC; 2009. p. 31-60.

28. Queiroz MV, Brito LM, Pennafot VP, Bezerra FS. Sensibilizando a criança com diabetes para o cuidado de si: contribuição à prática educativa. Esc Anna Nery. 2016;20(2):337-43.

29. Cassarino-Perez L, Alves CF, Dell'Aglio DD. Suporte social em adolescentes com diabete melito tipo I: uma revisão sistemática. Rev SPAGESP. 2014;15(1):33-48.

30. Moura DJ, Moura NS, Menezes LC, Barros AA, Guedes MV. Construção de cartilha sobre insulinoterapia para crianças com diabetes mellitus tipo 1. Rev Bras Enferm. 2017; 70 (1):7-14.

31. Czerwinski GP, Cogo AL. Webquest e blog como estratégias educativas em saúde escolar. Rev Gaúcha Enferm. 2018;39(0):e2017-0054. 